\title{
Dosing Accuracy of Direct Oral Anticoagulants in an Academic Medical Center
}

\author{
Janice B. Schwartz, MD ${ }^{1,2 \star}$, Steve Merrill, PharmD ${ }^{3,4}$, Noelle de Leon, PharmD, \\ Ashley Thompson, PharmD ${ }^{4,5}$, Margaret C. Fang, MD, $\mathrm{MPH}^{1}$
}

\begin{abstract}
${ }^{1}$ Department of Medicine and ${ }^{2}$ Department of Bioengineering and Therapeutic Sciences, University of California, San Francisco, California; ${ }^{3}$ Department of Clinical Sciences, College of Pharmacy, Touro University California, Vallejo, California; ${ }^{4}$ University of California San Francisco Medical Center, San Francisco, California; ${ }^{5}$ Department of Clinical Pharmacy, University of California, San Francisco, California.
\end{abstract}

BACKGROUND/OBJECTIVE: Direct-acting oral anticoagulants (DOACs) are increasingly used to prevent or treat thromboembolism. We conducted a study to compare how well initial DOAC prescribing for adult inpatients adhered to dosing recommendations approved by the US Food and Drug Administration (FDA).

DESIGN: Retrospective analysis.

SETTING: Single academic medical center, July 1, 2014 to June 30, 2015.

PATIENTS: 508 adult inpatients.

MEASUREMENTS: DOAC prescriptions were evaluated to determine whether they met FDA-recommended dosing and administration according to patient age, weight, sex, race, kidney function, diagnoses, and concomitant medications.

RESULTS: DOACs were prescribed in 635 admissions (247 apixaban, 97 dabigatran, 291 rivaroxaban). The indication was atrial fibrillation/flutter in 465 admissions (8\% with bioprostheses or valve repair), chronic deep vein thrombosis (DVT) in 67, acute DVT in 32, chronic pulmonary embolism in 23, acute pulmonary embolism in 19, DVT prevention after hip or knee surgery in 19, and non-FDA-approved indications in 10. Sixteen percent of orders for venous thromboembolic disease were for patients with active malignancy. Dosages not concordant with recommendations were prescribed for apixaban in $18 \%$ of admissions, for rivaroxaban in 14\%, and for dabigatran in 7\% $(P$ $=0.04)$. Lower than recommended dosing was more common than higher than recommended dosing $(P<0.05)$. Half the deviations were continuations of outpatient dosing. Atrial fibrillation/flutter and post-hip or -knee surgery dosing deviations were more common than venous thromboembolic disease deviations $(P<0.001)$ but were not related to prescriber specialty.

CONCLUSIONS: DOAC prescribing recommendation deviations that can affect clinical efficacy were identified. Education and point-of-care decision support tools for improving dosing are needed, as are outcome data for patients who receive DOACs at lower than recommended dosing or for off-label indications. Journal of Hospital Medicine 2017;12:544-550. (C) 2017 Society of Hospital Medicine
Direct-acting oral anticoagulants (DOACs) have been introduced into clinical use for stroke prevention in patients with nonvalvular atrial fibrillation (NVAF), prevention of venous thrombosis after hip or knee surgery, and treatment of deep vein thrombosis (DVT) and pulmonary embolism (PE). ${ }^{1-7}$ Advantages of DOACs over warfarin are often stated as fixed dosing, minor drug and food interactions, wider therapeutic index, and no need for laboratory test monitoring. ${ }^{1,8}$ Yet, recommended DOAC dosages vary by renal function and therapeutic indications. Dosing recommendations for prevention of stroke in patients with NVAF are based on estimated creatinine clearance (dabigatran, rivaroxaban, edoxaban), age (apixaban), weight (apixaban, edoxaban), serum creatinine level (apixaban, edoxaban), and presence of cirrhosis by Child-Pugh class ${ }^{9,10}$ (apixaban, edoxaban).4-6,11,12 Dosing recommendations based on coadminis-

\footnotetext{
*Address for correspondence and reprint requests: Janice B. Schwartz, MD, 302 Silver Ave, San Francisco, CA 94112; Telephone: 415-406-1573; Fax: 415-406-1577; E-mail: janice.schwartz@ucsf.edu

Received: October 31, 2016; Revised: December 16, 2016; Accepted: December 21, 2016
}

2017 Society of Hospital Medicine DOI 10.12788/jhm.2769 tration of strong CYP34A and P-glycoprotein inhibitors or inducers vary by DOAC. In addition, dabigatran cannot be crushed and must be stored in its original packaging, and rivaroxaban should be taken with food when the dose is over $10 \mathrm{mg}$.

We studied DOAC prescribing in adults admitted to a large academic medical center by comparing initial prescribed dosing with FDA-approved prescribing information. We hypothesized that the complexity of DOAC dosing may not be recognized by prescribers.

\section{METHODS}

Our study protocol was approved by the Committee on $\mathrm{Hu}$ man Research (Institutional Review Board) of the University of California San Francisco.

\section{Data Collection}

We used electronic medical records (EMRs) to identify adult inpatients who were prescribed a DOAC (apixaban, dabigatran, edoxaban, or rivaroxaban) at the University of California San Francisco Medical Center, a large academic hospital, between July 1, 2014 and June 30, 2015. Demographic and medical information related to therapeutic indications, contraindications, and indications for dose adjustments 
were collected and included diagnoses classified by International Classification of Diseases, Ninth Revision (ICD-9) and Tenth Revision (ICD-10) for venous thromboses; phlebitis or thrombophlebitis; PE or venous embolism; atrial arrhythmias; surgical procedures; cirrhosis and/or ascites or liver disease; coagulopathies; artificial heart valves or implanted devices; prior use of medications including parenteral anticoagulants; and laboratory data obtained before the first DOAC order (serum creatinine level, estimated glomerular filtration rate [eGFR] determined by Chronic Kidney Disease Epidemiology Collaboration, ${ }^{13}$ international normalized ratio, or, if available, activated partial thromboplastin time and bilirubin level). Creatinine clearance was calculated with the Cockcroft-Gault method ${ }^{14}$ using total body weight, per drug label recommendation. Child-Pugh class was calculated if cirrhosis was diagnosed. ${ }^{10}$ DOAC dose, frequency, dosing directions, and prescriber medical specialty were determined.

Accuracy of search results was confirmed by review of the first 200 patients' records. Records were manually reviewed for encounters lacking ICD-9/10 codes and approved DOAC indications $(30 \%)$ and encounters having multiple coded diagnostic indications (to identify the indication). ICD-9 codes for venous thrombosis were reviewed to differentiate acute from chronic events.

\section{Data Analysis}

The main outcome was concordance or discordance between the first DOAC prescribing order and the FDA-approved prescribing information at the time. Initial classification, performed by 2 independent reviewers (a pharmacist and a physician, or 2 pharmacists), was followed by adjudication and individual record review (by 2 independent reviewers) of all initial prescribing orders classified as discordant. A third reviewer adjudicated any disagreement. Records and notes were reviewed to identify stated or potential reasons for dosing variation and pre-admission prescriptions. Data are presented as means and standard deviations (SDs) and as raw numbers and percentages. Differences in patient characteristics by DOAC or therapeutic indication were determined by analysis of variance (ANOVA) with Bonferroni correction for post hoc comparisons. Dosing information was categorized as the same as recommended, lower than recommended, higher than recommended, or avoid drug use (drug-drug or drug-disease interaction), per FDA-approved prescribing information, and $\chi^{2}$ tests were used to determine whether variation in dosing occurred by individual DOAC, therapeutic indication, or prescriber specialty. Relationships between dosing variation and age or renal function were tested by ANOVA

Height, cm
TABLE 1. Patient Demographics

\begin{tabular}{lcccc}
\hline Demographic & Total & Apixaban & Dabigatran & Rivaroxaban \\
\hline Patients, n & 508 & 191 & 75 & 242 \\
\hline Sex, $\mathrm{n}$ & & & & \\
$\quad$ Male & 286 & 102 & 51 & 133 \\
Female & 222 & 89 & 24 & 109 \\
\hline Race, $\mathrm{n}$ & & & & \\
Black & 34 & 13 & 2 & 19 \\
White & 316 & 111 & 49 & 156 \\
Asian & 67 & 36 & 10 & 21 \\
Native American or Pacific Islander & 10 & 5 & 1 & 4 \\
Other & 61 & 21 & 9 & 31 \\
Unknown & 19 & 5 & 3 & 11
\end{tabular}

\begin{tabular}{lcccc}
\hline Ethnicity, n & & & & \\
$\quad$ Hispanic & 45 & 17 & 6 & 22 \\
Non-Hispanic & 445 & 168 & 68 & 209 \\
Unknown & 18 & 6 & 1 & 11 \\
\hline Age, y & & & & \\
Mean & 68.6 & 72.2 & 70.2 & 65.2 \\
SD & 14.7 & 13.8 & 12.0 & 15.3 \\
Range & $19-98$ & $19-98$ & $33-94$ & $20-97$ \\
\hline
\end{tabular}

\begin{tabular}{lcccc}
\hline Weight, $\mathrm{kg}$ & & & & \\
$\quad$ Mean & 82.9 & 78.8 & 86.8 & 84.9 \\
SD & 24.8 & 25.2 & 24.3 & 24.4 \\
Range & $36.4-225.5$ & $36.4-179.8$ & $49.1-156.8$ & $39.3-225.5$ \\
\hline
\end{tabular}

\begin{tabular}{lcccc} 
Mean & 170.5 & 169.5 & 173.2 & 170.4 \\
SD & 11 & 10.3 & 10.0 & 11.6 \\
Range & $143.5-203.2$ & $143.5-195.6$ & $152.4-198.1$ & $147.3-203.2$ \\
\hline Body mass index, kg/m & & & & \\
$\quad$ Mean & 28.4 & 27.2 & 28.7 & 29.2 \\
SD & 7.2 & 6.8 & 7.3 & 7.2 \\
Range & $14.4-71.3$ & $14.8-50.9$ & $17.6-59.0$ & $14.4-71.3$ \\
\hline
\end{tabular}

Creatinine level, $\mathrm{mg} / \mathrm{dL}$

$\begin{array}{lllll}\text { Mean } & 1.1 & 1.1 & 1.0 & 1.0\end{array}$

$\begin{array}{lllll}\text { SD } & 0.7 & 1.0 & 0.4 & 0.3\end{array}$

$\begin{array}{lllll}\text { Range } & 0.3-10.9 & 0.4-10.9 & 0.4-2.4 & 0.3-2.3\end{array}$

Estimated creatinine clearance, ${ }^{\mathrm{a}} \mathrm{mL} / \mathrm{min}$

\begin{tabular}{lcccc} 
Mean & 86.9 & 77.2 & 88.7 & 94.1 \\
SD & 48.8 & 50.0 & 48.0 & 47.3 \\
Range & $4-297$ & $4-297$ & $18-261$ & $24-267$ \\
\hline
\end{tabular}

aCalculated with Cockcroft-Gault method using total body weight.

NOTE: Significant differences were detected between DOAC groups for age (rivaroxaban vs apixaban or dabigatran, $P<0.02$ ), weight (rivaroxaban vs apixaban, $P<0.02$ ), body mass index (rivaroxaban vs apixaban, $P<0.02$ ), and creatinine or estimated creatinine clearance (apixaban vs rivaroxaban) by analysis of variance post hoc Bonferroni-Dunn method. No significant differences between sex or race proportions for DOAC groups were detected. Abbreviations: DOAC, direct-acting oral anticoagulant; SD, standard deviation.

with Bonferroni correction for post hoc comparisons.

\section{RESULTS}

There were 635 admissions with apixaban, dabigatran, or rivaroxaban prescribed for 508 patients (Table 1). Edoxaban was not on the formulary and not prescribed during the period studied. The therapeutic indication was prevention of embolic stroke in patients with atrial fibrillation/flutter or AF (465 admissions, or $73 \%$, with valvular disease and/ 
or tissue valve in 35), chronic DVT (67 admissions, or $11 \%$, with active malignancy in 14 ), acute DVT (32 admissions, with malignancy in 2), chronic PE (23 admissions, with malignancy in 3), acute PE (19 admissions, with malignancy in 4), and DVT prevention after hip or knee surgery (19 admissions). DOACs were prescribed for unapproved indications in 10 admissions, and these were excluded from further analysis (mural thrombus in 3 admissions, low ejection fraction in 2, bedrest immobilization in 2, aortic aneurysm in 1 , thrombocytosis in 1 , and extensive superficial venous thrombosis in 1) (Table 2).

Patients with AF were older with lower creatinine clearance compared to patients with other diagnoses. Mean (SD) patient age was 72.1 (12.7) years for AF, 53.1 (10.9) years for chronic PE, 55.5 (14) years for acute PE, 56.4 (15.9) years for chronic DVT, 57.9 (18.4) years for acute DVT, and 61.4 (11.6) years for DVT prevention after hip or knee surgery $(P<0.0001$ for all comparisons). Mean (SD) estimated creatinine clearance was $76.8(43.5) \mathrm{mL} / \mathrm{min}$ for AF, $92.4(44.4)$ $\mathrm{mL} / \mathrm{min}$ for DVT prevention after hip or knee surgery, $111(53) \mathrm{mL} / \mathrm{min}$ for chronic DVT, $118(55) \mathrm{mL} / \mathrm{min}$ for acute DVT, $126(60) \mathrm{mL} / \mathrm{min}$ for chronic PE, and 127 (54) $\mathrm{mL} / \mathrm{min}$ for acute PE $(P<0.0001$ for all comparisons). Differences between patient groups by therapeutic indication were not detected for weight, body mass index, or serum creatinine level.

The most frequent deviation from prescribing recommendations was omission of directions to administer rivaroxaban with food-93\% (248/268) of orders-but not for DVT prevention after hip or knee surgery, for which the 10-mg dose is appropriately administered without food. Doses were the same as recommended for $82 \%$ of apixaban orders, $84 \%$ of rivaroxaban orders, and $93 \%$ of initial dabigatran orders $(P<0.05$ for differences; Table 3). Dosages not concordant with FDA recommendations were prescribed in $44(18.1 \%)$ of 243 apixaban orders, $41(14.3 \%)$ of 286 rivaroxaban orders, and $7(7.2 \%)$ of 89 initial dabigatran orders. Lower than recommended doses were more common than higher than recommended doses (Table 3, Figure 1): 15.2\% versus $2.1 \%$ of apixaban orders, $9.4 \%$ versus $3.5 \%$ of rivaroxaban orders, and $4.2 \%$ versus $1.0 \%$ of initial dabigatran orders $(P<0.05)$.
TABLE 2. Treatment and Therapeutic Indications and Prescriber Specialties by Admission

\begin{tabular}{lcccc}
\hline & Total & Apixaban & Dabigatran & Rivaroxaban \\
\hline Treatment Indication & & & & \\
\hline Initial DOAC orders, $n$ (\% row) & 635 & $247(38.9 \%)$ & $97(15.3 \%)$ & $291(45.8 \%)$ \\
\hline $\begin{array}{l}\text { With }>1 \text { admission } \\
n(\% \text { row) }\end{array}$ & 96 & $44(45.8 \%)$ & $13(13.5 \%)$ & $39(40.6 \%)$ \\
$\%$ column & $15.1 \%$ & $17.8 \%$ & $13.4 \%$ & $13.4 \%$ \\
\hline
\end{tabular}

Therapeutic Indication

\begin{tabular}{|c|c|c|c|c|}
\hline \multicolumn{5}{|c|}{ Atrial fibrillation/flutter (valvular disease, $n=35$ ) } \\
\hline $\mathrm{n}(\%$ row $)$ & 465 & $224(48.2 \%)$ & $71(15.3 \%)$ & $170(36.6 \%)$ \\
\hline$\%$ column & $73.2 \%$ & $90.7 \%$ & $73.2 \%$ & $58.4 \%$ \\
\hline \multicolumn{5}{|c|}{ Acute PE (active malignancy, $n=4$ ) } \\
\hline $\mathrm{n}(\%$ row $)$ & 19 & $3(15.8 \%)$ & 0 & $16(84 \%)$ \\
\hline$\%$ column & $3.0 \%$ & $1.2 \%$ & 0 & $5.6 \%$ \\
\hline \multicolumn{5}{|c|}{ Chronic PE (active malignancy, $\mathrm{n}=3$ ) } \\
\hline n (\% row) & 23 & $1(4.3 \%)$ & $2(8.7 \%)$ & $20(87.0 \%)$ \\
\hline$\%$ column & $3.6 \%$ & $0.4 \%$ & $2.1 \%$ & $7.3 \%$ \\
\hline \multicolumn{5}{|c|}{ Acute DVT (active malignancy, $\mathrm{n}=$ = ) } \\
\hline n (\% row) & 32 & $7(21.8 \%)$ & $6(18.8 \%)$ & $19(59.4 \%)$ \\
\hline$\%$ column & $5.0 \%$ & $2.8 \%$ & $6.3 \%$ & $6.5 \%$ \\
\hline \multicolumn{5}{|c|}{ Chronic DVT (active malignancy, $n=14$ ) } \\
\hline $\mathrm{n}(\%$ row) & 67 & $7(10.4 \%)$ & $17(25.4 \%)$ & $42(62.7 \%)$ \\
\hline$\%$ column & $10.6 \%$ & $2.9 \%$ & $17.7 \%$ & $14.6 \%$ \\
\hline \multicolumn{5}{|c|}{ DVT prevention (hip or knee surgery) } \\
\hline $\mathrm{n}(\%$ row $)$ & 19 & $1(5.3 \%)$ & 0 & $18(94.7 \%)$ \\
\hline$\%$ column & $3.0 \%$ & $0.4 \%$ & 0 & $6.2 \%$ \\
\hline \multicolumn{5}{|c|}{ Unapproved indication ${ }^{\mathrm{a}}$} \\
\hline $\mathrm{n}(\%$ row $)$ & 10 & 4 & 1 & 5 \\
\hline$\%$ column & $1.6 \%$ & $1.6 \%$ & $1.0 \%$ & $2.1 \%$ \\
\hline
\end{tabular}

Prescriber Specialty

\begin{tabular}{|c|c|c|c|c|}
\hline \multicolumn{5}{|l|}{ Cardiology } \\
\hline n (\% row) & 202 & 113 (55.9\%) & 22 (10.9\%) & $67(33.2 \%)$ \\
\hline$\%$ column & $31.7 \%$ & $45.7 \%$ & $22.7 \%$ & $22.9 \%$ \\
\hline \multicolumn{5}{|c|}{ Emergency medicine } \\
\hline n (\% row) & 60 & $34(56.7 \%)$ & $1(3.3 \%)$ & $24(40 \%)$ \\
\hline$\%$ column & $9.4 \%$ & $13.8 \%$ & $2.1 \%$ & $8.2 \%$ \\
\hline \multicolumn{5}{|c|}{ Hospital medicine } \\
\hline n (\% row) & 131 & $35(26.7 \%)$ & 26 (19.8\%) & $70(53.4 \%)$ \\
\hline$\%$ column & $20.6 \%$ & $14.2 \%$ & $26.8 \%$ & $24.1 \%$ \\
\hline \multicolumn{5}{|c|}{ Other medical specialty } \\
\hline n (\% row) & 64 & $19(29.7 \%)$ & $16(25.0 \%)$ & $29(45.3 \%)$ \\
\hline$\%$ column & $10.1 \%$ & $7.7 \%$ & $16.5 \%$ & $10.0 \%$ \\
\hline \multicolumn{5}{|l|}{ Surgery } \\
\hline n (\% row) & 132 & $41(31.8 \%)$ & $28(21.2 \%)$ & $63(47.7 \%)$ \\
\hline$\%$ column & $20.7 \%$ & $16.6 \%$ & $28.9 \%$ & $21.6 \%$ \\
\hline \multicolumn{5}{|c|}{ Orthopedic surgery } \\
\hline n (\% row) & 47 & 6 (12.8\%) & $3(6.4 \%)$ & $38(80.9 \%)$ \\
\hline$\%$ column & $7.4 \%$ & $2.4 \%$ & $3.1 \%$ & $13.1 \%$ \\
\hline
\end{tabular}

aMural thrombus in 3 patients, low ejection fraction in 2, immobilization in 2, thrombocytosis in 1, aortic aneurysm in 1, and superficial venous thrombosis in 1 .

NOTE: Abbreviations: DOAC, direct-acting oral anticoagulant; DVT, deep vein thrombosis; PE, pulmonary embolism. 


\begin{tabular}{|c|c|c|c|c|c|}
\hline & \multirow[b]{2}{*}{$\mathrm{n}$} & \multicolumn{4}{|c|}{ Dosing vs Prescribing Recommendation, n (\%) } \\
\hline & & Same As & Higher Than & Lower Than & Avoid Use \\
\hline \multicolumn{6}{|l|}{ Direct-Acting Oral Anticoagulant ${ }^{\mathrm{a}}$} \\
\hline Apixaban & 243 & $199(81.9)$ & $5(2.1)$ & 37 (15.2) & $2(0.8)$ \\
\hline Dabigatran & 96 & $89(92.7)$ & $1(1.0)$ & $4(4.2)$ & $2(2.1)$ \\
\hline Rivaroxaban & 286 & $245(85.7)$ & $10(3.5)$ & $27(9.4)$ & $4(1.4)$ \\
\hline \multicolumn{6}{|l|}{ Therapeutic Indication ${ }^{\mathrm{b}}$} \\
\hline Atrial fibrillation/flutter & 465 & $384(82.6)$ & $14(3.0)$ & $63(13.5)$ & $4(0.1)$ \\
\hline Acute PE & 19 & $18(94.7)$ & $0(0)$ & $1(5.3)$ & $0(0)$ \\
\hline Chronic PE & 23 & $20(87.0)$ & $0(0)$ & $1(4.3)$ & $2(8.7)$ \\
\hline Acute DVT & 32 & $29(90.6)$ & $0(0)$ & $2(6.3)$ & $1(3.1)$ \\
\hline Chronic DVT & 67 & $66(98.5)$ & $0(0)$ & $1(1.5)$ & $0(0)$ \\
\hline DVT prevention (hip or knee surgery) & 19 & $16(84.2)$ & $2(10.5)$ & $0(0)$ & $1(5.3)$ \\
\hline
\end{tabular}

Failure to avoid drug use (for potential drug-drug or drugdisease interactions) was uncommon (1\%-2\%). There were more deviations from recommended doses for patients with AF or DVT prevention after hip or knee surgery than for patients with acute or chronic PE or acute DVT (Table 3). No significant differences were detected between prescribed and recommended doses by prescriber specialty.

In most cases, a reason for deviating from FDA dosing recommendations was not stated in the EMR. The exception was fluctuating renal function, which was cited in 8 cases.

For apixaban, patients who were prescribed lower than recommended doses were older than those prescribed recommended doses: mean (SD), 78.1 (12.2) years versus 71 (13.6) years $(P=0.003)$. Seventy-six percent of those prescribed lower than recommended doses were older than 75 . Prescriptions for apixaban at lower than recommended doses

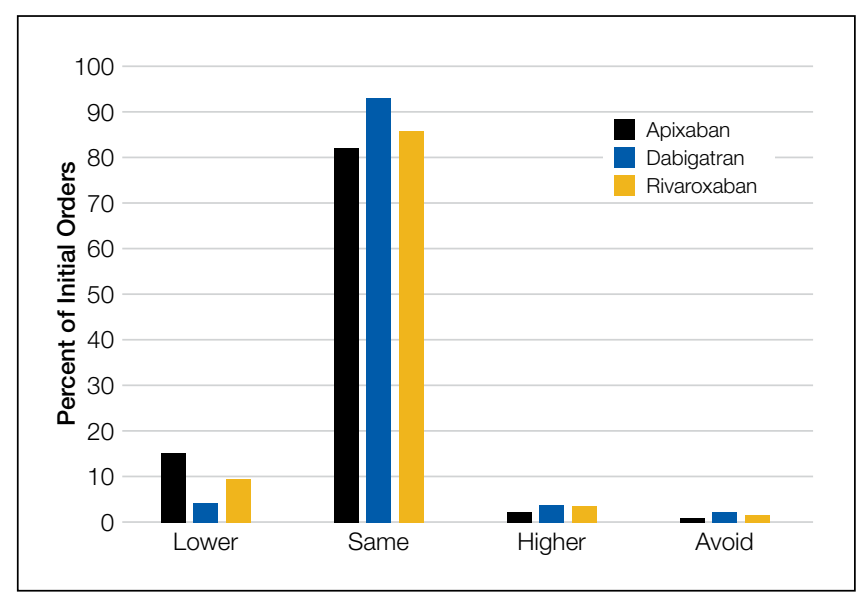

FIG. Comparison of initial direct-acting oral anticoagulant dosing with FDA-recommended dosing.

NOTE: Percentage of initial orders for apixaban, dabigatran, and rivaroxaban, categorized as same as recommended, lower than recommended, higher than recommended, and avoid drug use, per FDA-approved prescribing information. Abbreviation: FDA, US Food and Drug Administration. were continuations of prior outpatient prescriptions in 20 of 37 cases (almost half), and in 12 cases (one-fourth) antiplatelet drugs were coprescribed (aspirin in 10 cases, clopidogrel in 1, prasugrel in 1). For rivaroxaban, older age was associated with both lower than recommended dosing $(P=$ $0.003)$ and higher than recommended dosing $(P<0.001)$. Variations from prescribing recommendations were continuations of outpatient rivaroxaban doses in about two-thirds (26 of $41 ; 63.4 \%$ ) with 13 receiving antiplatelet drugs. For dabigatran, 6 of 7 orders not in agreement with recommendations were continuations of outpatient dosing.

The specific equation used to estimate renal function also had the potential to lead to dosing errors. Among the 41 rivaroxaban patients categorized as receiving doses discordant with recommendations, 8 would have had an inappropriate DOAC dose if eGFR were used instead of eCrCL as recommended. No relationships were detected for other patient variables/ measures and dosing deviations from recommendations.

\section{DISCUSSION}

We examined initial hospital orders for DOACs in adults admitted to a single academic medical center during 20142015. Dabigatran, apixaban and rivaroxaban were prescribed for prevention of stroke in patients with atrial fibrillation/ flutter (AF) in three quarters of the encounters similar to national patterns. (15) Prescribing departures from FDA-approved recommendations ranged from failure to prescribe rivaroxaban with food to failure to recognize drug-drug interactions in $1 \%$ to $2 \%$. Unexpectedly, lower than recommended dosing was more common than higher than recommended dosing of the three DOACs.

Rivaroxaban bioavailability is dose dependent with the presence of food required to enhance absorption for doses over $10 \mathrm{mg}$ that are used for prevention of stroke in patients with non-valvular AF or treatment of DVT or PE. ${ }^{5,16}$ Peak rivaroxaban concentrations are $75 \%$ higher and the total 
area under the concentration vs. time curve after dosing is $40 \%$ higher when rivaroxaban is administered with high fat high calorie meals compared to the fasting state. ${ }^{16}$ If rivaroxaban is not administered with food, drug concentrations and pharmacologic effects may be less than in clinical trials that specified co-administration with food. ${ }^{17-19}$ A small survey of outpatients receiving rivaroxaban found that $23 \%$ reported taking it without food. ${ }^{20} \mathrm{With}$ electronic pharmacy systems in almost all hospitals and electronic prescriber order entry in most, automated addition of directions for rivaroxaban administration with food for doses over $10 \mathrm{mg}$ to labels or dispensing instructions could easily correct this deviation from recommended practice.

Lower than recommended doses were prescribed in $9.4 \%$ of orders for rivaroxaban and $15.2 \%$ of orders for apixaban, with dose-deviations often appearing to be a continuation of outpatient doses. Patients 75 years or older were more likely to receive lower than recommended dosing of apixaban. Reductions in apixaban doses from $5 \mathrm{mg}$ twice daily to $2.5 \mathrm{mg}$ twice daily are recommended in patients with non-valvular AF with two of the following criteria: age $\geq 80$ y, weight $\leq 60$ $\mathrm{kg}$, serum creatinine $\geq 1.5 \mathrm{mg} / \mathrm{dL}$ or co-administration of a strong $\mathrm{PgP}$ inhibitor to a patient without 2 of the 3 dose reduction criteria. Our study was not designed to determine reasons for under-dosing, but we speculate that clinicians may have considered patients aged $75-79$ years to be similar to those 80 years of age or older, or, older and not as healthy as those enrolled in randomized trials. ${ }^{21-25}$ The median age of our patients with $\mathrm{AF}$ receiving apixaban was $75 \mathrm{y}$ (interquartile range of 16) vs $70 y$ (interquartile range 63-76) in the pivotal trial comparing warfarin to apixaban. ${ }^{21}$ Renal function was also lower with $37 \%$ having eCrCL below $50 \mathrm{~mL} /$ min compared to $17 \%$ in ARISTOTLE. (21). Twenty-six percent of our apixaban-treated AF patients qualified for the lower $2.5 \mathrm{mg}$ twice daily compared to only $5 \%$ of ARISTOTLE participants, ${ }^{21}$ further suggesting differences between patients in our sample compared to randomized trial participants.

Concerns regarding bleeding or falls in older patients, may also have contributed to lower than recommended doses. Recent analyses of patients at risk for falls confirmed that increased risk of falling was associated with more bone fractures, bleeding and all-cause death but not stroke or systemic emboli, and with less severe bleeding with the DOAC edoxaban compared to warfarin. ${ }^{26}$ While a rationale for personalized or lower than recommended dosing of apixaban may exist in very old patients and those at risk of falls and bleeding, more data are needed to determine outcomes of lower than recommended doses of DOACs before such an approach can be endorsed. Monitoring of anticoagulant effect in patients who receive doses lower than those investigated in clinical trials could provide important information. The assays that measure DOAC effects are likely to be more available because of the use of reversal agents in the setting of bleeding with DOACs. ${ }^{27}$

We had anticipated higher than recommended dosing for rivaroxaban as recommendations are based on creatinine clearance while laboratories routinely report estimated glomerular filtration rate (eGFR) that can provide higher estimates of renal clearance and estimated DOAC doses in older and smaller individuals. ${ }^{28}$ Higher than recommended dosing was found in only $3.5 \%$ of our sample. In half, eGFR estimates were higher than creatinine clearance estimates. An international postmarketing registry of rivaroxaban use for the prevention of stroke in patients with NVAF, which included outpatients, found that $36 \%$ of those with creatinine clearances below $50 \mathrm{~mL} / \mathrm{min}$ received a dose higher than recommended, and $15 \%$ received a dose lower than expected. ${ }^{29}$ A more recent outpatient registry report on patients with NVAF, in which apixaban, dabigatran, or rivaroxaban was administered, found that overall $9.4 \%$ received a dose lower than recommended, and $3.4 \%$ were overdosed, with a similar percentage $(34 \%)$ of rivaroxaban patients with creatinine clearance of 15 to $50 \mathrm{~mL} / \mathrm{min}$ receiving higher than recommended dosing. ${ }^{30}$ The lower rate of higher-than-recommended doses that we observed may have been related to the routine measurement of serum creatinine and attention to dosing adjustments for renal function in the inpatient setting compared to the outpatient setting. In addition, renal function data may not be available to outpatient pharmacies, limiting potential input on dosing recommendations. At least one cardiac society recommends monitoring of renal function in patients treated with DOACs, annually in patients with normal estimated creatinine clearance and more frequently (at intervals in months equal to the creatinine clearance divided by 10) in patients with abnormal creatinine clearance. ${ }^{11}$ A hospital encounter provides an opportunity to assess or reassess renal status to optimize DOAC dosing.

Dabigatran was the first DOAC introduced into use in the United States with the same dose recommended for prevention of stroke in patients with $\mathrm{AF}$ or venous thromboembolic disease with reductions for creatinine clearance below 30 $\mathrm{mL} / \mathrm{min}$ or creatinine clearance between 30 and $50 \mathrm{~mL} / \mathrm{min}$ and concomitant use of the potent P-glycoprotein inhibitor dronedarone or systemic ketoconazole. The relative simplicity of dosing may have been responsible for the lowest rate of prescribing outside of recommendations observed in this study, but the low dabigatran use limits analyses of contributing factors.

Failure to avoid drug use in combination with use of strong $\mathrm{P}$-glycoprotein inducers or inhibitors was infrequent but should be preventable. Current prescribing recommendations refer to "strong" P-glycoprotein inhibitors and list different specific agents that interact with each DOAC without a standardized definition or classification. Standardized classifications or reference sources would be helpful.

Our primary goal in this study was to compare initial prescribed dosing of DOACs with FDA-approved prescribing directions. However, therapeutic indication data warrant discussion. In our sample, $7.5 \%$ of patients with AF had bioprosthetic valves or recent mitral valve repair or replacement. 
Using the NVAF definition found in the 2014 AHA/ACC/ HRS (American Heart Association, American College of Cardiology, Heart Rhythm Society) AF guidelines'- "absence of rheumatic mitral valve disease, a prosthetic heart valve, or mitral valve repair"- these patients would not appear to be candidates for DOACs. However, arguments have been made that a bioprosthetic heart valve or native valve after valve repair does not have a risk profile for thromboembolism that differs from other forms of NVAF and would be equally responsive to DOAC therapy. ${ }^{31}$ Data are sparse, but retrospective subanalyses of limited numbers of patients with valvular disease (including bioprosthesis and mitral repair patients but excluding mechanical valve patients) enrolled in the pivotal DOAC studies support this conclusion. ${ }^{32}$ For the first months after biological valve replacement (including catheter-based valve replacement), recent European guidelines recommend vitamin $\mathrm{K}$ antagonists but also state, "NOACs probably deliver the same protection." ${ }^{8}$ DOACs were also used for management of venous thromboembolic disease (both acute and chronic) in patients with active cancer. Our data predate the most recent American College of Chest Physician guidelines on treatment of venous thromboembolism in patients with cancer, which provide grade $2 \mathrm{~B}$ recommendations for use of low-molecular-weight heparin (LMWH) over vitamin $\mathrm{K}$ antagonists and grade $2 \mathrm{C}$ recommendations for use of LMWH over dabigatran, rivaroxaban, apixaban, or edoxaban. ${ }^{33}$

Our study had several limitations. First, data were from a single US academic medical center, though similar rates of prescribing deviation from recommendations have been reported for rivaroxaban and dabigatran in NVAF patients in other countries. ${ }^{29,34}$ Second, therapeutic indications may have been misclassified because of errors, incomplete EMR data, or multiple indications. Third, we analyzed the first DOAC order and not dispensing information or subsequent corrections. Therefore, deviations from recommendations should not be interpreted as errors that reached patients. We evaluated dosing based on the measures used at the time of hospital admission, noting that, in a significant fraction of deviations from recommended doses, they represented continuations of outpatient doses when renal function or weight may have differed, and it is unknown whether patients were counseled to take rivaroxaban with food in the outpatient setting. Fourth, the number of patients with acute DVT was small, so firm conclusions cannot be drawn for this specific population. Fifth, our estimates of off-label dosing may have been underestimates, as data on cancer and cancer activity or cardiac valvular disease may not have been complete.

\section{CONCLUSION}

Healthcare professionals are prescribing DOACs in ways that differ from recommendations. These differences may reflect the older ages and reduced renal function of clinical populations relative to randomized clinical trial groups, but they could also potentially alter clinical efficacy. Our findings support the need to evaluate the appropriateness and dosing of DOACs at each encounter and to determine the outcomes of patients treated with lower than recommended doses of DOACs and the outcomes of DOAC-treated patients with bioprostheses or active malignancies.

\section{Acknowledgment}

The authors thank Tobias Schmelzinger for electronic data extraction and compilation and University of California San Francisco students Eduardo De La Torre Cruz (School of Pharmacy) and Carlos Mikell (School of Medicine) for assistance with data review.

Disclosure: Dr. Schwartz reports receiving personal fees from Bristol-Myers Squibb and Amgen and grants from Bristol-Myers Squibb and Pfizer, outside the submitted work. The other authors have nothing to report.

\section{References}

1. January CT, Wann LS, Alpert JS, et al. 2014 AHA/ACC/HRS guideline for the management of patients with atrial fibrillation: executive summary. A report of the American College of Cardiology/American Heart Association Task Force on Practice Guidelines and the Heart Rhythm Society. J Am Coll Cardiol. 2014;64(21):2246-2280.

2. Saraf K, Morris PD, Garg P, Sheridan P, Storey R. Non-vitamin K antagonist oral anticoagulants (NOACs): clinical evidence and therapeutic considerations. Postgrad Med J. 2014;90(1067):520-528.

3. Yeh CH, Gross PL, Weitz JI. Evolving use of new oral anticoagulants for treatment of venous thromboembolism. Blood. 2014;124(7):1020-1028.

4. Pradaxa website. https://www.pradaxa.com. Accessed June 1, 2017.

5. Xarelto website. https://www.xarelto-us.com. Accessed June 1, 2017.

6. Eliquis website. http://www.eliquis.com. Accessed June 1, 2017.

7. Savaysa [prescribing information]. Tokyo, Japan: Daiichi Sankyo; 2015.

8. Kirchhof P, Benussi S, Kotecha D, et al. 2016 ESC guidelines for the management of atrial fibrillation developed in collaboration with EACTS. Eur Heart J. 2016;37(38):2893-2962.

9. Child C, Turcotte J. Surgery and portal hypertension. In: Child CG, ed. The Liver and Portal Hypertension. Philadelphia, PA: Saunders; 1964:50-64.

10. Pugh RN, Murray-Lyon IM, Dawson JL, Pietroni MC, Williams R. Transection of the oesophagus for bleeding oesophageal varices. Br J Surg. 1973;60(8):646-649.

11. Heidbuchel H, Verhamme P, Alings M, et al. Updated European Heart Rhythm Association practical guide on the use of non-vitamin $\mathrm{K}$ antagonist anticoagulants in patients with non-valvular atrial fibrillation. Europace. 2015;17(10):1467-1507.

12. Savaysa website. https://savaysahcp.com. Accessed June 1, 2017.

13. Levey AS, Stevens LA, Schmid CH, et al; CKD-EPI (Chronic Kidney Disease Epidemiology Collaboration). A new equation to estimate glomerular filtration rate. Ann Intern Med. 2009;150(9):604-612.

14. Cockcroft DW, Gault MH. Prediction of creatinine clearance from serum creatinine. Nephron. 1976;16(1):31-41.

15. Rose AJ, Reisman JI, Allen AL, Miller DR. Potentially inappropriate prescribing of direct-acting oral anticoagulants in the Veterans Health Administration. Am J Pharm Benefits. 2016;4(4):e75-e80.

16. Stampfuss J, Kubitza D, Becka M, Mueck W. The effect of food on the absorption and pharmacokinetics of rivaroxaban. Int J Clin Pharmacol Ther. 2013;51(7):549-561.

17. Patel MR, Mahaffey KW, Garg J, et al; ROCKET AF Investigators. Rivaroxaban versus warfarin in nonvalvular atrial fibrillation. N Engl J Med. 2011;365(10):883-891.

18. EINSTEIN Investigators, Bauersachs R, Berkowitz SD, et al. Oral rivaroxaban for symptomatic venous thromboembolism. N Engl J Med. 2010;363(26):2499-2510.

19. EINSTEIN-PE Investigators, Büller HR, Prins MH, et al. Oral rivaroxaban for the treatment of symptomatic pulmonary embolism. N Engl J Med. 2012;366(14):1287-1297.

20. Simon J, Hawes E, Deyo Z, Bryant-Shilliday B. Evaluation of prescribing and patient use of target-specific oral anticoagulants in the outpatient setting. J Clin Pharm Ther. 2015;40(5):525-530.

21. Granger CB, Alexander JH, McMurray JJ, et al; ARISTOTLE Committees and Investigators. Apixaban versus warfarin in patients with atrial fibrillation. $N$ Engl J Med. 2011;365(11):981-992.

22. Ruff CT, Giugliano RP, Braunwald E, et al. Comparison of the efficacy and safety of new oral anticoagulants with warfarin in patients with atrial fibrillation: a meta-analysis of randomised trials. Lancet. 2014;383(9921):955-962.

23. van der Hulle T, Kooiman J, den Exter PL, Dekkers OM, Klok FA, Huisman MV. Effectiveness and safety of novel oral anticoagulants as compared with vitamin $\mathrm{K}$ antagonists in the treatment of acute symptomatic venous thromboembolism: a sys- 
tematic review and meta-analysis. J Thromb Haemost. 2014;12(3):320-328.

24. Schuh T, Reichardt B, Finsterer J, Stöllberger C. Age-dependency of prescribing patterns of oral anticoagulant drugs in Austria during 2011-2014. J Thromb Thrombolysis. 2016;42(3):447-451.

25. Stöllberger C, Brooks R, Finsterer J, Pachofszky T. Use of direct-acting oral anticoagulants in nonagenarians: a call for more data. Drugs Aging. 2016;33(5):315-320.

26. Steffel J, Giugliano RP, Braunwald E, et al. Edoxaban versus warfarin in atrial fibrillation patients at risk of falling: ENGAGE AF-TIMI 48 analysis. J Am Coll Cardiol. 2016;68(11):1169-1178.

27. Ruff CT, Giugliano RP, Antman EM. Management of bleeding with non-vitamin $\mathrm{K}$ antagonist oral anticoagulants in the era of specific reversal agents. Circulation. 2016;134(3):248-261.

28. Schwartz JB. Potential impact of substituting estimated glomerular filtration rate for estimated creatinine clearance for dosing of direct oral anticoagulants. J Am Geriatr Soc. 2016;64(10):1996-2002.
29. Camm AJ, Amarenco P, Haas S, et al; XANTUS Investigators. XANTUS: a real-world, prospective, observational study of patients treated with rivaroxaban for stroke prevention in atrial fibrillation. Eur Heart J. 2016;37(14):1145-1153.

30. Steinberg BA, Shrader P, Thomas L, et al; ORBIT-AF Investigators and Patients. Off-label dosing of non-vitamin $\mathrm{K}$ antagonist oral anticoagulants and adverse outcomes: the ORBIT-AF II Registry. J Am Coll Cardiol. 2016;68(24):2597-2604.

31. Fauchier L, Philippart R, Clementy N, et al. How to define valvular atrial fibrillation? Arch Cardiovasc Dis. 2015;108(10):530-539.

32. Di Biase L. Use of direct oral anticoagulants in patients with atrial fibrillation and valvular heart lesions. J Am Heart Assoc. 2016;5(2)

33. Kearon C, Akl EA, Ornelas J, et al. Antithrombotic therapy for VTE disease: CHEST guideline and expert panel report. Chest. 2016;149(2):315-352.

34. Larock AS, Mullier F, Sennesael AL, et al. Appropriateness of prescribing dabigatran etexilate and rivaroxaban in patients with nonvalvular atrial fibrillation: a prospective study. Ann Pharmacother. 2014;48(10):1258-1268. 